\title{
Efeitos da fotobioestimulação e estimulação elétrica na cicatrização e qualidade de vida de indivíduos com úlceras cutâneas: terapias isoladas e associadas
}

\section{Effects of photobiostimulation and electric stimulation in wound healing and quality of life of individuals with skin ulcers: isolated and associated therapies}

\author{
Carla Roberta Nunes Polachini, Viviane Bohrer Berni, Jéssica Michelon Bellé, \\ Daiane Flores de Oliveira, Michele Forgiarini Saccol
}

\section{Como citar este artigo:} POLACHINI, CARLA R. N.; BERNI, VIVIANE B.; BELLÉ, JÉSSICA M.; OLIVEIRA, DAIANE F.; SACCOL, MICHELE F; Efeitos da fotobioestimulação e estimulação elétrica na cicatrização e qualidade de vida de indivíduos com úlceras cutâneas: terapias isoladas e associadas. Revista Saúde (Sta. Maria). 2019; 45 (3).

\section{Autor correspondente: \\ Nome: Carla Roberta Nunes Polachini E-mail: polachini.carla@gmail.com Telefone: (55) 999087517 \\ Formação Profissional: Doutora em Ciências Biológicas - Bioquímica Toxicológica pela Universidade Federal de Santa Maria (UFSM), Santa Maria, RS, Brasil.}

Filiação Institucional: Universidade Federal de Santa Maria

Endereço para correspondência: Avenida Roraima n: 1000

Bairro: Camobi

Cidade: Santa Maria

Estado: Rio Grande do Sul

CEP: 97 105-900

Data de Submissão:

23/08/2019

Data de aceite:

18/12/2019

Conflito de Interesse: Não há conflito de interesse

\section{(cc) $\mathrm{BY}-\mathrm{NC}-\mathrm{ND}$}

\section{RESUMO}

Objetivo: investigar os efeitos de intervenções fisioterapêuticas isoladas e associadas com laser de baixa potência e microcorrente no reparo cicatricial e qualidade de vida em indivíduos com úlceras cutâneas de diferentes etiologias. Métodos: Estudo quase-experimental realizado no Hospital Universitário de Santa Maria. A amostra constituiu-se de nove pacientes com úlceras cutâneas. Esses foram divididos em três grupos: laser ( $n=3$ ), microcorrente $(n=3)$ e laser associado a microcorrente $(n=3)$, com aplicação de laser $(660 \mathrm{~nm}$; modo contínuo; $P=30 \mathrm{~mW}$; densidade de energia $\left.=4 \mathrm{~J} / \mathrm{cm}^{2}\right)$ e microcorrente $(f=130 \mathrm{~Hz} ; i=300 \mu \mathrm{A} ; \mathrm{t}=30$ minutos), totalizando 15 sessões de fisioterapia em torno de 45 dias (maio a julho). Avaliou-se a área da úlcera cutânea e processo de cicatrização (uso de régua associada a fotogrametria), dor (Escala Visual analógica), além de sensibilidade (por meio de estesiômetro). Também, foi aplicado o questionário de Qualidade de Vida WHOQOL-bref. Todas essas variáveis e análises ocorreram em três momentos (Pré, $10^{\mathrm{a}}$ e $15^{\mathrm{a}}$ intervenção). Resultados: Houve uma redução significativa no comprimento da úlcera cutânea, verificou-se no pré $2,80(1,45-4,72) \mathrm{cm}$ e na $15^{\mathrm{a}}$ aplicações 2,10 $(0-4,27) \mathrm{cm}(P=0,001)$, bem como a largura também reduziu na $15^{\mathrm{a}}$ intervenção $(P=0,001)$. Assim, a área da ferida apresentou uma significante redução na $15^{\mathrm{a}}$ intervenção, de $17,8(5,70-30,76) \mathrm{cm}^{2}$ para $7,17(0-21,30) \mathrm{cm}^{2}$ $(P=0,002)$. Além disso, constatou-se uma diferença significativa na qualidade de vida dos pacientes já a partir da $10^{\mathrm{a}}$ sessão $(\mathrm{P}<0,05)$. Conclusões: $\mathrm{O}$ uso da fotobioestimulação e da microcorrente aplicados de forma isolada e associada otimizaram o reparo tecidual das úlceras cutâneas de diversas etiologias promovendo uma redução na área da ferida com consequente melhora na cicatrização e na qualidade de vida dos pacientes. Nesse contexto, esta pesquisa agrega valor à prática clínica fisioterapêutica no tratamento para feridas.

PALAVRAS-CHAVE: Úlcera Cutânea, Modalidades de Fisioterapia, Terapia a Laser de Baixa Potência, Estimulação Elétrica, Cicatrização.

\section{ABSTRACT}

Objective: To investigate the effects of isolated and associated physical therapy interventions with low-level laser therapy and microcurrent on repair healing and quality of life in individuals with skin ulcers of different etiologies. Methods: Quasi-experimental study performed from the Federal University of Santa Maria Hospital. The sample consisted of nine patients with cutaneous ulcers. These individuals were divided into three groups: laser $(n=3)$, microcurrent $(n=3)$ and laser associated with microcurrent $(n=3)$, with laser application (660nm; continuous mode; $P=30 \mathrm{~mW}$; energy density $=4 \mathrm{~J} / \mathrm{cm}^{2}$ ) and microcurrent ( $f=130 \mathrm{~Hz} ; i=300 \mu \mathrm{A} ; \mathrm{t}=30$ minutes), totaling 15 physiotherapy sessions around 45 days (May to July). We evaluated skin ulcer area and healing process (by ruler associated with photogrammetry, pain (Analog Visual Scale) as well as sensitivity (by esthesiometer) Also, we applied the Quality of Life questionnaire (WHOQOL-bref). All these variables and analysis was made in three moments (Pre, 10th and 15th intervention). Results: There was a significant reduction in the length of the cutaneous ulcer, before $2.80(1.45-4.72) \mathrm{cm}$ and in the 15th applications $2.10(0-4.27) \mathrm{cm}(P=0.001)$, results also showed a reduction in width in the 15th intervention $(P=0.001)$. Thus, we found a significant reduction in wound area in the 15th intervention, from $17.8(5.70-30.76) \mathrm{cm}^{2}$ to $7.17(0-21.30) \mathrm{cm}^{2}(P=0.002)$. In addition, it was revealed a significant difference in the quality of life of patients already as from 10th session $(P<0.05)$. Conclusions: The use of photobiostimulation and microcurrent applied alone and combined optimized the tissue repair of skin ulcers of various etiologies and, consequently, to provide a better quality of life for patients as well as to reduce in the wound area by improvement in healing. In this context, this research adds value to the physical therapy clinical practice in the treatment of wounds.

KEYWORDS: Skin Ulcer, Physical Therapy Modalities, Low-Level Light Therapy, Electric Stimulation, Wound Healing 


\section{INTRODUÇÃO}

As úlceras cutâneas são definidas como a perda da continuidade ou rupturas da integridade estrutural e funcional da pele devido a danos físico-químicos, lesões térmicas ou oriundas de doenças ${ }^{1}$. Essas lesões constituem um problema de saúde para o paciente bem como à sociedade, uma vez que são acompanhadas de significativas repercussões clínicas, pessoais e econômicas ${ }^{2}$. Considerando-se que estão associadas a limitações físicas, mentais e sociais decorrentes da dor, problemas motores e sensitivos, infecções e hospitalização prolongada além de, afastamento do convívio em sociedade, por conseguinte afetam a qualidade de vida e interação social dos sujeitos acometidos ${ }^{3,4}$.

A etiologia dessas lesões é multifatorial podendo ser oriundas de complicações cirúrgicas, queimaduras, traumas, resultante de pressão ou cisalhamento, ou ainda devido a condições subjacentes, como doenças imunológicas, metabólicas, insuficiência venosa e/ou arterial|3,5. Essas feridas são um grande desafio terapêutico em todo mundo, estimando-se que o número de casos de indivíduos que sofrem com essas lesões deve aumentar à medida que a população envelhecer e pela a maior incidência dos fatores de risco, dentre eles, o diabetes mellitus (DM), o tabagismo, obesidade e distúrbios vasculares ${ }^{1,6}$.

As estratégias de terapêuticas disponíveis para as úlceras cutâneas abrange uma diversidade de medidas podendo essas serem invasivas e/ou não invasivas ${ }^{6}$. Assim, dentre os métodos de tratamento não-invasivos tem sido observado na prática clínica dos fisioterapeutas a utilização do laser de baixa potência bem como da microcorrente, tendo em vista que esses agentes físicos promovem a aceleração do processo cicatricial atuando na sequência de eventos fisiológicos e bioquímicos como a inflamação, síntese de colágeno, formação do tecido de granulação e reepitelização ${ }^{7}$. Ao estimular eventos biológicos como nutrição, proliferação celular e controle dos processos inflamatórios e infecciosos favorece-se a reparação tecidual ${ }^{8}$.

A fotobiomodulação promovida pelo laser consegue estimular a função mitocondrial quando a luz é absorvida pelo citocromo c oxidase, desse modo é um recurso capaz de promover a regeneração tecidual, redução da dor e edema, e ainda auxilia no desempenho muscular ${ }^{9}$. Logo, o laser de baixa potência e intensidade destaca-se como um meio terapêutico importante na cicatrização de feridas e alívio do quadro álgico, uma vez que essa opção de tratamento gera diversos efeitos biológicos promovendo o aumento do fluxo sanguíneo, redução da inflamação local, além de melhora na função imunológica ${ }^{10}$.

Destaca-se também, o uso da estimulação elétrica por meio da microcorrente, uma corrente subsensorial, que não causa dor e/ou desconforto, de baixo custo e não invasiva ${ }^{7}$. Esse recurso utiliza correntes elétricas semelhantes as produzidas pelo organismo durante a cicatrização tecidual sendo considerada de grande utilidade e benéfica ao tratamento de lesões ${ }^{11,12}$. Desse modo, o objetivo deste estudo foi investigar 0 efeito de terapias isoladas e associadas do laser de baixa potência e microcorrente no reparo cicatricial e qualidade de vida em indivíduos com úlceras cutâneas de diferentes etiologias. 


\section{MÉTODO}

Trata-se de um estudo quantitativo, de natureza aplicada, do tipo quase-experimental ${ }^{13}$, realizado no Hospital Universitário de Santa Maria (HUSM), da Universidade Federal de Santa Maria, no período de maio a julho de 2019. Em todas as etapas do estudo, foram respeitados os princípios éticos estabelecidos na Resolução n 466/2012 do Conselho Nacional de Saúde. Foi aprovado pelo Comitê de Ética em Pesquisa da Instituição (nº 07931219.4.0000.5346).

\section{Participantes}

A amostra por conveniência compreendeu 9 pacientes com úlcera cutânea de diferentes etiologias, de ambos os sexos, com idade superior a 21 anos, sob tratamento clínico baseado em curativos (medidas gerais de higienização da úlcera com soro fisiológico, coberturas de alta e baixa tecnologia conforme indicação do caso, oclusão com gaze e enfaixamento por meio de atadura, a fim de evitar interferência externa no processo cicatricial). Esses participantes foram alocados em três grupos mediante sorteio, o Grupo Laser (GL): tratados com laser de baixa potência $(n=3)$, o Grupo Microcorrente (GM): tratados com microcorrente ( $n=3$ ) e o Grupo Laser e Microcorrente (GLM): tratados com laser associado a microcorrente $(n=3)$.

Não foram inclusos no estudo os pacientes que apresentavam marcapasso, osteomielite, úlceras cutâneas há mais de cinco anos, em tratamento de câncer nos últimos 6 meses, uso de próteses metálicas próximas a área da aplicação do recurso, bem como intolerância ao posicionamento necessário para a aplicação dos recursos. Esses critérios levam em consideração algumas contraindicações quanto ao uso da corrente elétrica, o risco de disseminação da afecção e o bem-estar do participante, priorizando-se a segurança do paciente.

\section{Procedimentos}

Inicialmente, os pacientes foram submetidos a uma ficha de avaliação para coleta de dados pessoais e clínicos com a finalidade de realizar a anamnese e o exame físico, por meio de identificação. Em seguida, na forma de entrevista, foi aplicado o Questionário World Health Organization Quality of Life (WHOQOL-bref). Após, realizou-se as fotos bem como a avaliação da dor e a mensuração da sensibilidade próximo a área da úlcera cutânea. Os participantes foram avaliados e tratados individualmente e todos os testes foram aplicados pelo mesmo examinador. Todos os procedimentos foram descritos de modo mais abrangente na sequência (subitem avaliações).

As avaliações foram realizadas em três períodos (pré-intervenção, $10^{\mathrm{a}}$ aplicação e ao término do protocolo estabelecido, que corresponde a $15^{\mathrm{a}}$ aplicação). No total foram realizadas 15 sessões de tratamento fisioterapêutico, durante o período de aproximadamente 1 mês e meio, sendo os atendimentos efetuados 2 vezes por semana. Todavia, 0 participante 3 (na $4^{\mathrm{a}}$ aplicação), o participante 7 (na 12a aplicação) e o participante 8 (na $7^{\mathrm{a}}$ aplicação) não conseguiram 
deslocar-se até o HUSM por dificuldade de transporte, assim acabaram realizando apenas um atendimento na respectiva semana, entretanto, todos os participantes totalizaram 15 intervenções.

\section{Avaliações}

\section{Área da úlcera cutânea}

A mensuração foi mediante fotogrametria e uso de régua de papel após debridamento realizando-se a medida bidimensional para o acompanhamento da evolução da cicatrização ${ }^{14,15}$. 0 registro fotográfico foi padronizado utilizando-se uma câmera traseira dupla de 12MP+5MP (smartphone Motorola ${ }^{\circledR}$ moto G6), posicionada a aproximadamente $35 \mathrm{~cm}$ da úlcera, perpendicularmente, contendo na imagem uma régua em contato com a pele, com escala em centímetro verificando (comprimento x largura).

Dor

A Escala Visual Analógica (EVA) foi utilizada para avaliar a percepção da intensidade da dor por parte do paciente. AEVA é uma escala constituída por uma linha de $10 \mathrm{~cm}$ que tem, em geral, como extremos as frases verbais indicando "sem dor" a $0 \mathrm{~cm}$ e "dor intensa" a $10 \mathrm{~cm}$ relatada pelo sujeito ${ }^{16}$.

\section{$\underline{\text { Sensibilidade }}$}

A sensibilidade tátil foi verificada mediante uso do Estesiômetro (modelo de bolso - "Sensikit") que consiste em um conjunto de seis monofilamentos (Semmes-Weinstein) de nylon, de comprimentos iguais, cores e diâmetros diferentes, que exercem forças de $0,05 \mathrm{~g}$ a $300 \mathrm{~g}$. As respectivas cores e valores de força de cada filamento são: verde $(0,05 \mathrm{~g})$, azul $(0,2 \mathrm{~g})$, violeta $(2,0 \mathrm{~g})$, vermelho $(4,0 \mathrm{~g})$, laranja $(10,0 \mathrm{~g})$ e rosa $(300,0 \mathrm{~g})$. 0 paciente relata quando sentir a aplicação, a qual ocorre de forma crescente na região ao redor da úlcera.

\section{Qualidade de vida}

Foi aplicado o Questionário WHOQOL-bref como um instrumento de avaliação da qualidade de vida. Esse é uma versão abreviada do WHOQOL 100. É constituído por 26 questões das quais duas são questões gerais de qualidade de vida e 24 são distribuídas em quatro domínios: físico; psicológico; relações sociais e meio ambiente. Os resultados foram avaliados mediante a atribuição de escores para cada questão, os quais foram transformados numa escala de zero a 100, onde zero corresponderá a uma pior qualidade de vida e valores mais próximo de 100 significa uma melhor qualidade de vida ${ }^{17,18}$. 


\section{Intervenções}

Os pacientes foram submetidos a terapia apenas com laser, microcorrente e associados. As intervenções foram realizadas com o paciente posicionado em um decúbito que permitiu manter a área da lesão exposta, após a assepsia do local com solução fisiológica a 0,9\% e gaze estéril bem como debridamento da lesão antes da aplicação do laser. Ao término de cada aplicação foi realizado o tratamento clínico baseado em curativos pela equipe de enfermagem.

No GL utilizou-se o aparelho de laser da marca IBRAMED® modelo Laserpulse Diamond Line, com as seguintes características: Laser visível, alumínio-gálio-índio-fósforo (AIGalnP) na faixa de 660nm, modo contínuo, potência de $30 \mathrm{~mW}$ e densidade de energia de $4 \mathrm{~J} / \mathrm{cm}^{2}$. A aplicação foi realizada por meio da técnica pontual, ao redor da lesão, sem contato direto (distância aproximada de $1 \mathrm{~mm}$ entre a ponta da caneta com plástico filme e a ferida), com aproximadamente $1 \mathrm{~cm}$ de distância entre os pontos. A caneta foi posicionada perpendicularmente à lesão e a quantidade de pontos foi estabelecida de acordo com o tamanho da úlcera ${ }^{19}$.

No GM foi usado o aparelho da marca IBRAMED® modelo Ruby Line, com 4 canais. 0 equipamento foi programado na terapia por microcorrentes, modo contínuo, frequência de $130 \mathrm{~Hz}$, intensidade 300 microampères durante 30 minutos $^{20}$. Eletrodos autoadesivos e hipoalergênicos (Carciß) de $5 \times 5 \mathrm{~cm}$ foram posicionados de forma cruzada nas extremidades da lesão. Cabe destacar que cada participante possuia o seu kit de eletrodos.

Para o GLM a aplicação da terapia com laser e microcorrente ocorreu em dias alternados, desse modo utilizou-se somente um dos equipamentos a cada dia de intervenção, e os parâmetros mantiveram-se os mesmos.

O tratamento proposto foi realizado por uma profissional fisioterapeuta com experiência em lesões de pele e duas acadêmicas do curso de fisioterapia que foram devidamente treinadas para aplicação dos recursos eletrotermofoterapêuticos pela fisioterapeuta experiente na área.

\section{Análise estatística}

Os dados são apresentados pela média \pm erro padrão da média ou em mediana e amplitude interquartil (p2575), bem como em frequências absoluta e relativa (\%). Para a análise estatística dos resultados obtidos utilizou-se o programa GraphPad Prism (GraphPad Software, San Diego, CA, versão 5.0). As variáveis foram submetidas ao teste de normalidade (Kolmogorov-Smirnov). Após, foi utilizado o teste paramétrico ANOVA (análise de variância) para medidas repetidas seguido do teste de post hoc de Bonferroni e os dados com distribuição assimétrica foram analisados pelo teste de Friedman. As diferenças foram consideradas estatisticamente significativas quando $P<0,05$. 


\section{RESULTADOS}

Foram selecionados 10 indivíduos, no entanto, um (1) foi excluído porque não compareceu durante duas semanas ao atendimento. A amostra final foi constituída por 9 voluntários, sendo 3 do sexo feminino e 6 do sexo masculino. A média de idade dos pacientes foi de $58( \pm 5,34)$ anos e $33,33 \%$ são diabéticos. Em relação ao tempo de surgimento ferida, a média foi de 10,77 ( $\pm 3,59)$ meses e as etiologias diferenciadas de acordo com o perfil da amostra. A caracterização geral dos participantes da pesquisa foram demonstradas na Tabela 1.

Tabela 1. Gênero, idade, etiologia e tempo da úlcera cutânea, presença do diabetes mellitus e cobertura usada no curativo dos participantes do estudo

\begin{tabular}{|c|c|c|c|c|c|c|}
\hline & $\begin{array}{c}\text { Gênero } \\
\text { (F/M) }\end{array}$ & $\begin{array}{l}\text { Idade } \\
\text { (anos) }\end{array}$ & Etiologia da Úlcera cutânea & $\begin{array}{l}\text { Tempo } \\
\text { da lesão } \\
\text { (meses) }\end{array}$ & $\begin{array}{l}\text { Diabético } \\
\text { (S/N) }\end{array}$ & $\begin{array}{l}\text { Cobertura utilizada } \\
\text { no curativo }\end{array}$ \\
\hline P1 & $\mathrm{F}$ & 65 & $\begin{array}{c}\text { Cicatrização por } 3^{a} \text { intenção após } \\
\text { cirurgia de hérnia inguinal } D\end{array}$ & $\begin{array}{l}2 \text { meses e } \\
\text { meio }\end{array}$ & $\mathrm{N}$ & $\begin{array}{l}\text { Hidrogel com } \\
\text { alginato }\end{array}$ \\
\hline P 2 & M & 53 & $\begin{array}{c}\text { Queimadura de } 2^{\circ} \text { grau profunda em } \\
\text { membro superior e hemitórax } D\end{array}$ & 4 meses & $\mathrm{N}$ & Hidrofibra de prata \\
\hline P 3 & $\mathrm{~F}$ & 77 & $\begin{array}{l}\text { Ferimento aberto em joelho D por } \\
\text { acidente de trânsito }\end{array}$ & 3 meses & $S$ & AGE \\
\hline P 4 & $\mathrm{~F}$ & 73 & $\begin{array}{c}\text { Sequela de hanseníase - alterações } \\
\text { vasculares na região anterior e lateral } \\
\text { da perna D }\end{array}$ & 24 meses & $\mathrm{N}$ & Papaína 6\% \\
\hline P 5 & M & 62 & Amputação tipo Lisfranc à D & 1 mês & $S$ & $\begin{array}{l}\text { Hidrofibrina com } \\
\text { prata }\end{array}$ \\
\hline P 6 & M & 71 & Sequela de osteomielite no pé $\mathrm{E}$ & 32 meses & $\mathrm{N}$ & $\begin{array}{l}\text { Hidrogel com } \\
\text { alginato }\end{array}$ \\
\hline P 7 & M & 24 & $\begin{array}{l}\text { Trauma pérfuro-cortante (calha de aço) } \\
\text { na região medial e posterior da perna } E\end{array}$ & 24 meses & $\mathrm{N}$ & Alginato de cálcio \\
\hline P 8 & M & 54 & $\begin{array}{l}\text { Lesão traumática (cerca de madeira) } \\
\text { próximo a região poplítea D }\end{array}$ & 2 meses & $\mathrm{N}$ & $\begin{array}{l}\text { Hidrogel com } \\
\text { alginato }\end{array}$ \\
\hline P 9 & M & 51 & Amputação tipo Lisfranc à D & $\begin{array}{l}4 \text { meses e } \\
\text { meio }\end{array}$ & $S$ & $\begin{array}{l}\text { Hidrogel com } \\
\text { alginato }\end{array}$ \\
\hline
\end{tabular}

P: participante; F: feminino; M: masculino; D: direita; E: esquerda; S: sim; N: não; Ácidos graxos essenciais: AGE.

A evolução da cicatrização das úlceras cutâneas de diferentes etiologias por meio da fotogrametria desde a avaliação inicial, na $10^{\mathrm{a}}$ e $15^{\mathrm{a}}$ intervenção, por meio do uso do laser, microcorrente (Figura 1a) e laser associado a microcorrente (Figura 1b). 
a

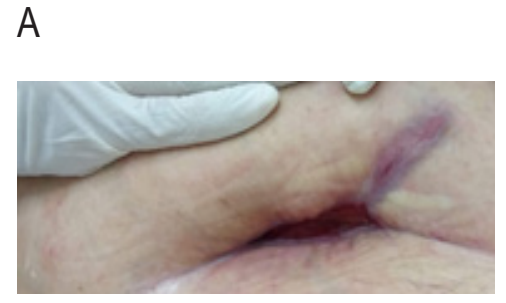

PRÉ-intervenção

B

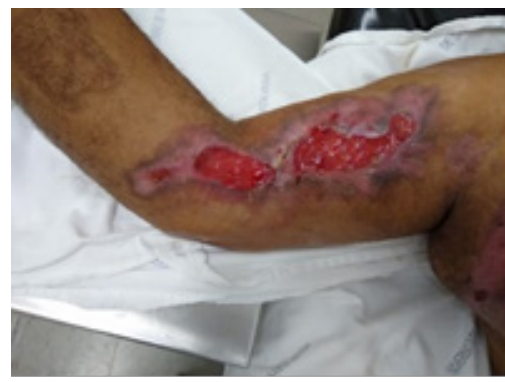

PRÉ-intervenção

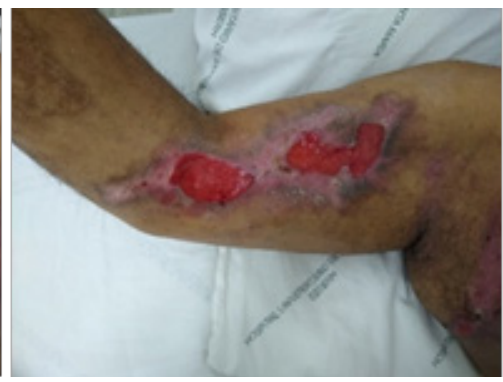

$10^{\mathrm{a}}$

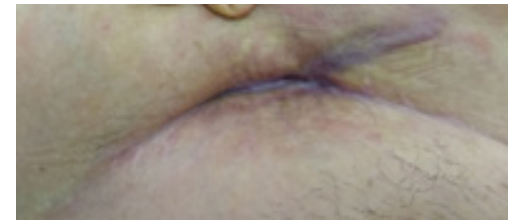

$10^{\mathrm{a}}$

C

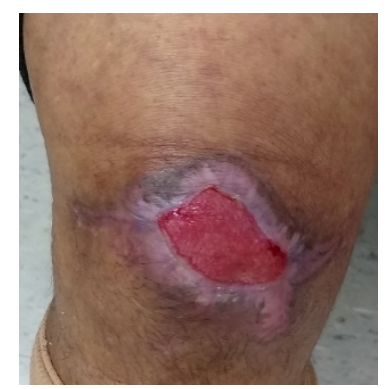

PRÉ-intervenção

D

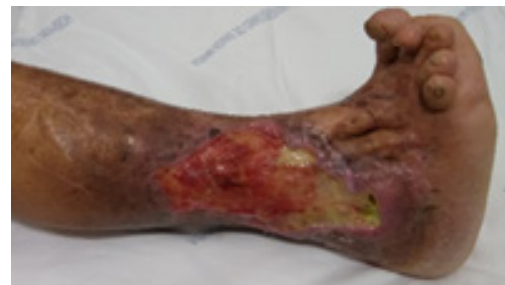

PRÉ-intervenção

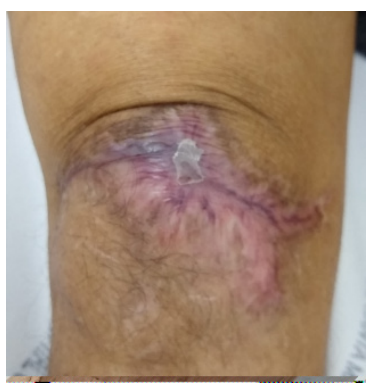

$10^{\mathrm{a}}$

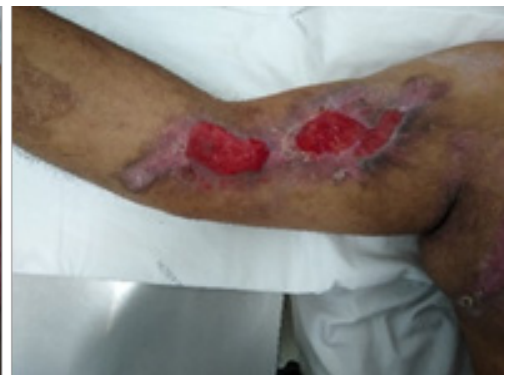

$15^{\mathrm{a}}$

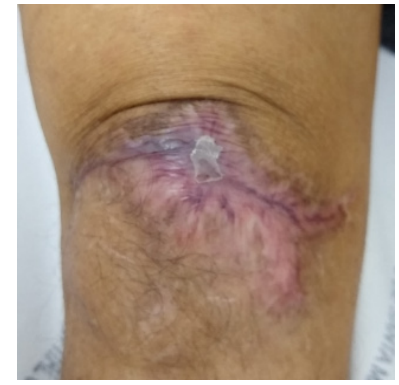

$15^{\mathrm{a}}$

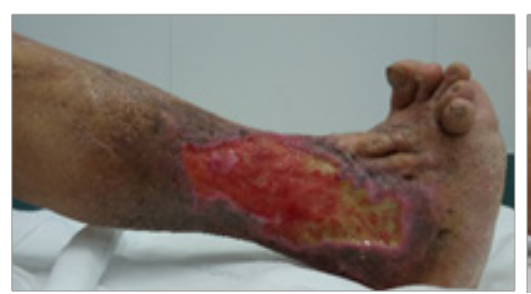

$10^{\mathrm{a}}$

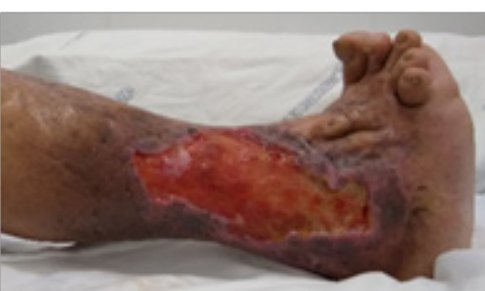

$15^{\mathrm{a}}$ 
E

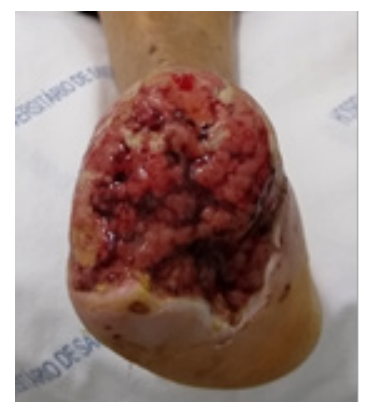

PRÉ-intervenção

F

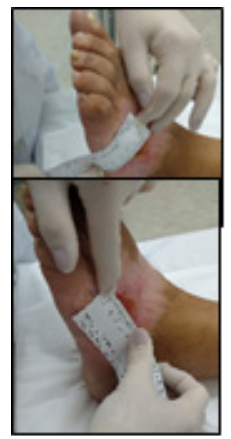

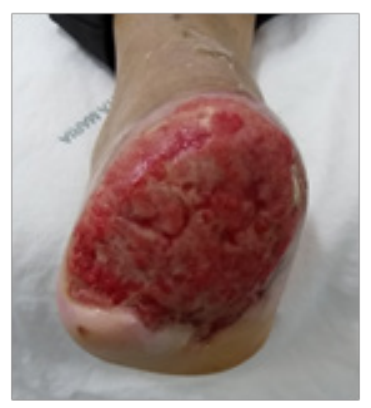

$10^{\mathrm{a}}$

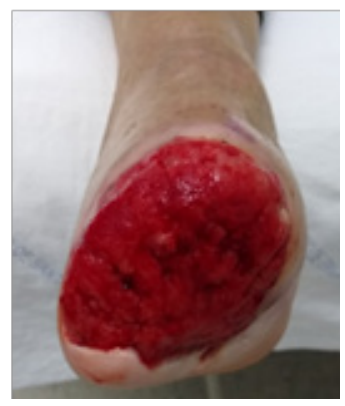

$15^{\mathrm{a}}$
PRÉ-intervenção

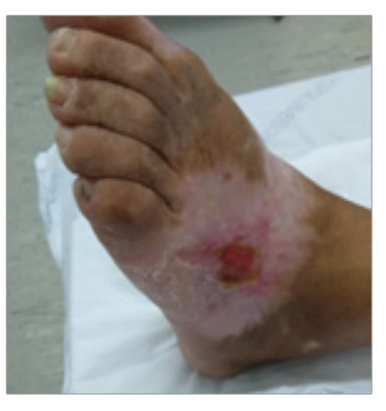

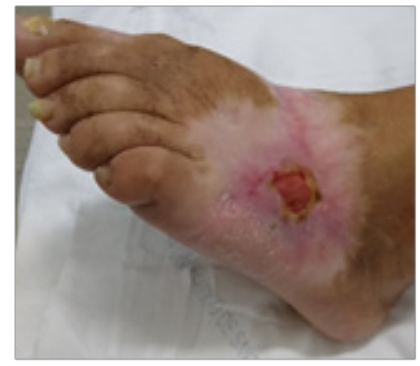

$10^{a}$

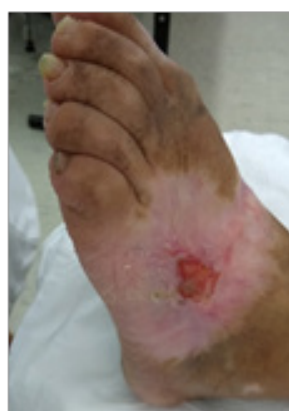

$15^{\mathrm{a}}$

b

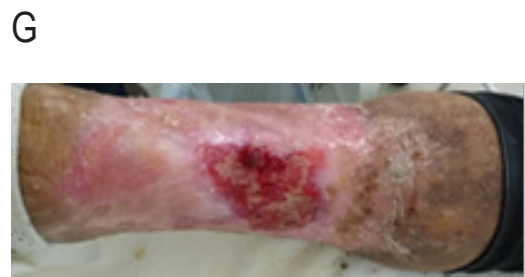

PRÉ-intervenção

$\mathrm{H}$

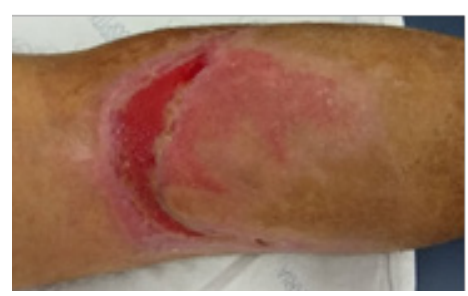

PRÉ-intervenção

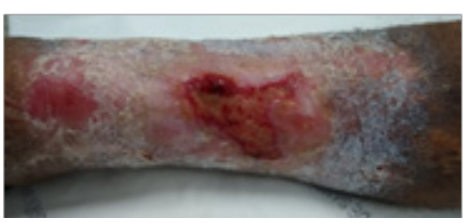

$10^{a}$

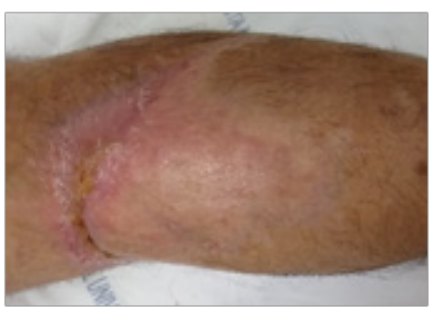

$10^{a}$

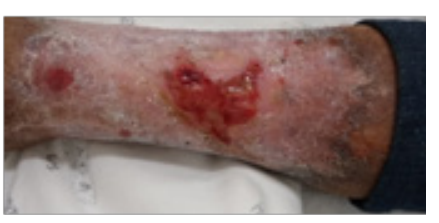

$15^{\mathrm{a}}$

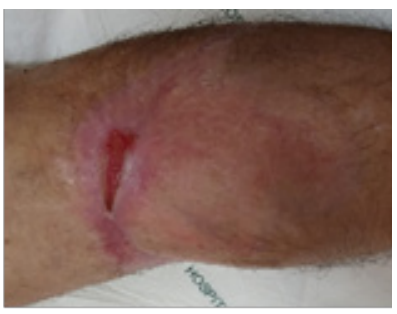

$15^{a}$ 


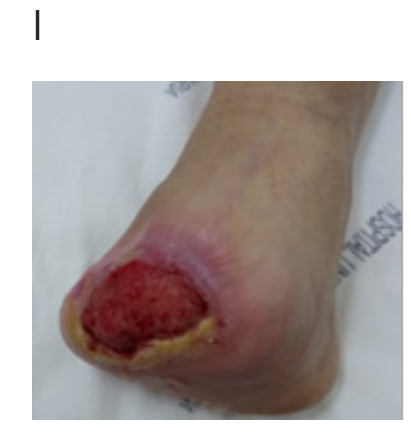

PRÉ-intervenção

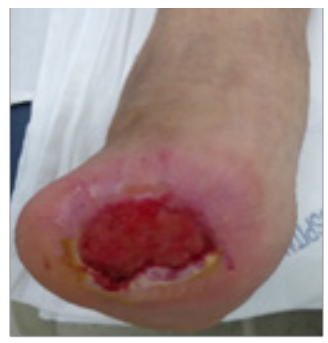

$10^{\mathrm{a}}$

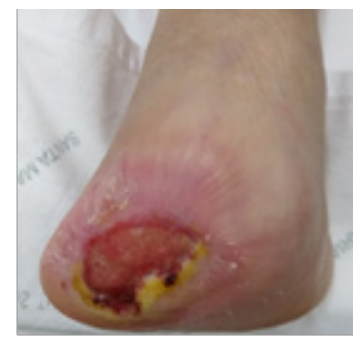

$15^{\mathrm{a}}$

Figura 1. Evoluções dos tratamentos. Participante 1 (A), 2 (B) e 3 (C) n=3, intervenção apenas com laser; Participante 4 (D), 5 (E) e $6(F) n=3$, intervenção apenas com microcorrente (a). Participante $7(G), 8(H)$ e $9(I) n=3$, tratamento com laser associado a microcorrente (b). \# Participante 6 (F) demonstra-se como eram realizadas as mensurações de comprimento e largura com uso da régua de papel descartável.

Em relação a área de cicatrização, observou-se redução no comprimento e na largura das feridas tanto nos pacientes tratados com as terapias isoladas como quando houve associação das mesmas (Tabela 2).

Tabela 2- Descrição dos resultados individuais encontrados antes, na $10^{\mathrm{a}}$ e $15^{\mathrm{a}}$ intervenção apenas com laser, microcorrente e associados.

\begin{tabular}{cccc}
\hline $\mathbf{P}$ & $\begin{array}{c}\text { Comprimento/largura }(\mathrm{cm}) \\
\text { PRÉ-intervenção }\end{array}$ & $\begin{array}{c}\text { Comprimento/largura }(\mathrm{cm}) \\
\text { intervenção } \mathbf{1 5}^{\mathbf{a}}\end{array}$ & $\begin{array}{c}\text { Comprimento/largura (cm) } \\
\text { intervenção 10 }\end{array}$ \\
\hline 1 & 1,2 / 6,5 & 0 & $0^{*}$ \\
2 & $1,0 / 1,6$ (I) & $1,0 / 1,6$ & 0 \\
& $3,4 / 9,5(\mathrm{II})$ & $3,0 / 7,2$ & $3,0 / 7,5$ \\
& $2,6 / 6,0$ (III) & $3,2 / 6,2$ & $3,0 / 5,9$ \\
3 & $0,8 / 1,0$ (IV) & 0 & 0 \\
4 & $3,0 / 5,0$ & $0,6 / 1,6$ & 0 \\
5 & $14,3 / 7,2$ & $14,3 / 7,3$ & $14,0 / 7,2$ \\
6 & $13 / 10,5$ & $12,0 / 9,0$ & $10,8 / 9,0$ \\
7 & $2,5 / 2,0$ & $2,2 / 1,8$ & $1,5 / 1,6$ \\
8 & $5,0 / 4,0$ & $5,0 / 3,6$ & $4,7 / 3,2$ \\
9 & $2,2 / 11,7$ & 0 & $1,0 / 3,0$ \\
\hline
\end{tabular}

$\mathrm{P}=$ participante. * Para o participante 1 as avaliações ocorreram até a $10^{\mathrm{a}}$ aplicação; I - V, referem-se a localização de cada uma das úlceras no mesmo participante sendo que (I)- hemitórax direito; (II)- braço; (III)- entre o braço e antebraço; (IV) - antebraço.

Ao se realizar a análise estatística considerando-se separadamente o comprimento e a largura da ferida, foi observada uma diferença significativa no comprimento das úlceras ao comparar os resultados da avaliação inicial 2,80 
$(1,45-4,72) \mathrm{cm}$ e $15^{\mathrm{a}}$ aplicação $2,10(0-4,27) \mathrm{cm}(\mathrm{P}=0,001)$. Também, verificou-se uma redução significativa na largura da ferida, tendo-se uma mediana de $5,80(2,50-8,92) \mathrm{cm}$ inicialmente, e $3,10(0-6,87) \mathrm{cm}$ ao final do tratamento com 15 intervenções dos recursos eletrotermofototerapêuticos ( $P=0,001)$ (Figura 2a e 2b).

a

\section{Comprimento}

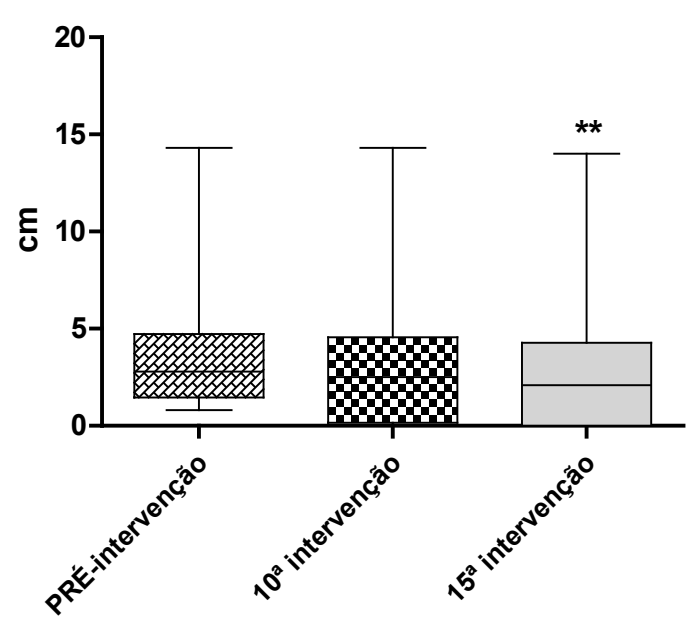

b

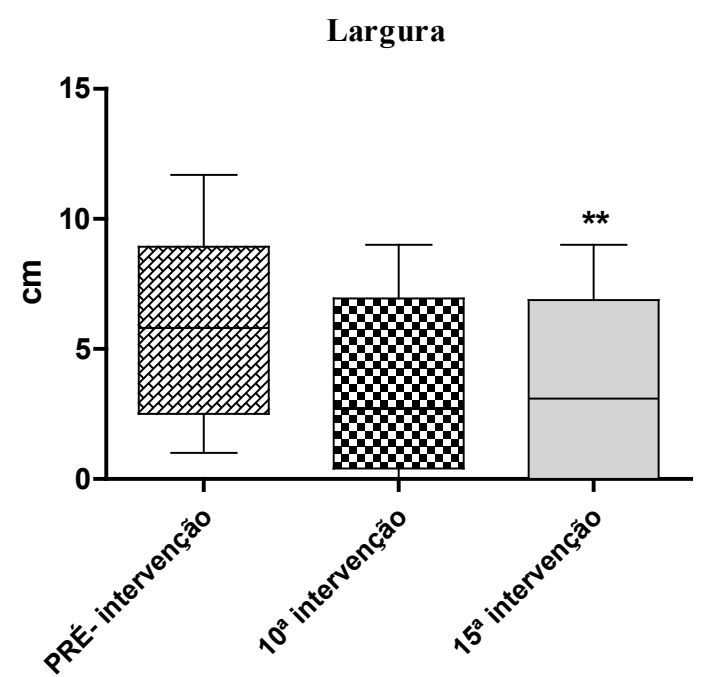

Figura 2- Medidas em comprimento e largura das úlceras cutâneas. Valores expressos em mediana \pm amplitude interquartil (p25-p75). * = diferença significativa em comparação a pré-intervenção $(P<0,05)$.

Em relação a área da úlcera cutânea, também foi observado uma redução significativa na $15^{a}$ intervenção, de $17,8(5,70-30,76) \mathrm{cm}^{2}$ para $7,17(0-21,30) \mathrm{cm}^{2}, \mathrm{P}=0,002$ (Figura 3).

\section{Área}

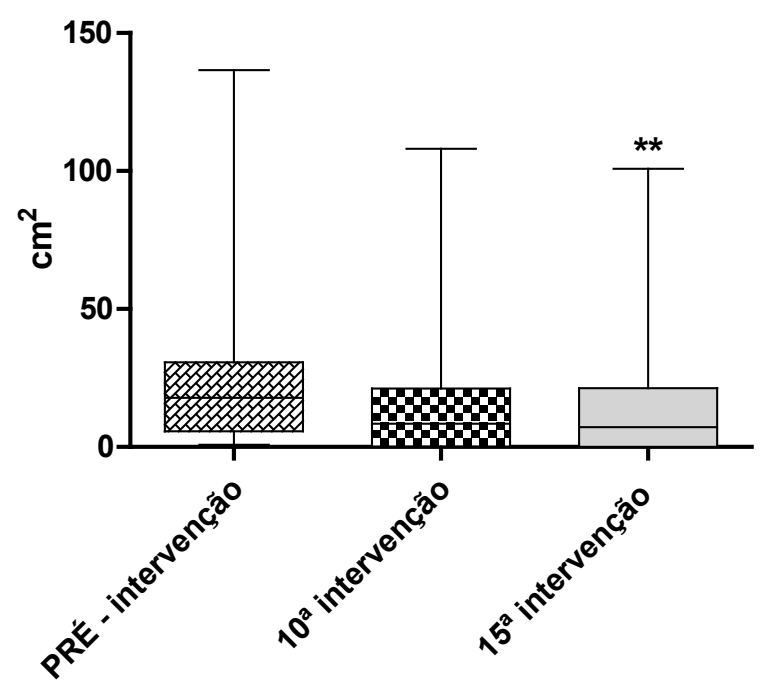

Figura 3- Área das úlceras cutâneas. Valores expressos em mediana \pm amplitude interquartil (p25-p75). * $=$ diferença significativa em comparação a pré-intervenção $(P<0,05)$. 
A tabela 3 descreve os resultados de dor e sensibilidade referida pelos pacientes com úlceras cutâneas de diversas etiologias. A partir das avaliações de percepção da dor, pode ser observado que, considerando os participantes do estudo que apresentavam dor na ferida, apenas o participante 1 não alterou a sua percepção do quadro álgico na $15^{\mathrm{a}}$ intervenção com a aplicação das terapias de forma isolada ou associada. Assim, os resultados indicam que 22,22\% (n total=9, $G L=3$ ), $22,22 \%$ ( $n$ total=9, $G M=3$ ), $11,11 \%$ ( $n$ total=9, $G L M=3$ ) apresentaram alteração no limiar de dor na $10^{a}$ e $15^{a}$ aplicação dos recursos. Já, em relação a análise sensibilidade próximo as bordas da lesão cutânea, 55,55\% da amostra apresentou modificações na $10^{\circledR}$ intervenção, e 66,66\% na última avaliação ( $15^{\mathrm{a}}$ intervenção) quando comparado com a avaliação inicial.

Tabela 3. Dados obtidos utilizando a Escala Visual Analógica (EVA) e o estesiômetro antes, na $10^{\mathrm{a}}$ e $15^{\mathrm{a}}$ aplicação de laser, microcorrente e associados.

\begin{tabular}{|c|c|c|c|c|c|c|}
\hline \multicolumn{2}{|l|}{ Variável } & \multirow{2}{*}{$\begin{array}{c}\text { PRÉ- } \\
\text { intervenção }\end{array}$} & \multirow{2}{*}{$\begin{array}{c}10^{\mathrm{a}} \\
\text { intervenção }\end{array}$} & \multirow{2}{*}{$\begin{array}{c}15^{\mathrm{a}} \\
\text { intervenção }\end{array}$} & \multirow[t]{2}{*}{ PRÉ x10a } & \multirow[t]{2}{*}{ PRÉ x15 } \\
\hline Dor & & & & & & \\
\hline & P1 & 2 & 2 & - & $=$ & - \\
\hline & P2 & 7 & 5 & 2 & $\downarrow$ & $\downarrow$ \\
\hline & P 3 & 5 & 2 & 0 & $\downarrow$ & $\downarrow$ \\
\hline & P 4 & 0 & 1 & 2 & $\uparrow$ & $\uparrow$ \\
\hline & P 5 & 1 & 1 & 0 & $=$ & $\downarrow$ \\
\hline & P 6 & 4 & 0 & 1 & $\downarrow$ & $\downarrow$ \\
\hline & P7 & 6 & 0 & 0 & $\downarrow$ & $\downarrow$ \\
\hline & P 8 & 0 & 0 & 0 & $=$ & $=$ \\
\hline & P 9 & 1 & 0 & 0 & $\downarrow$ & $\downarrow$ \\
\hline \multicolumn{7}{|c|}{ Sensibilidade } \\
\hline & P 1 & Laranja & Laranja & - & $=$ & $=$ \\
\hline & P2 & Azul & Verde & Verde & $\uparrow$ & $\uparrow$ \\
\hline & P3 & Laranja & Violeta & Azul & $\uparrow$ & $\uparrow$ \\
\hline & P 4 & Laranja & Azul & Verde & $\uparrow$ & $\uparrow$ \\
\hline & P 5 & Verde & Violeta & Azul & $\downarrow$ & $\downarrow$ \\
\hline & P 6 & Violeta & Violeta & Vermelho & $=$ & $\downarrow$ \\
\hline & P7 & Verde & Verde & Verde & $=$ & $=$ \\
\hline & P 8 & Violeta & Verde & Azul & $\uparrow$ & $\uparrow$ \\
\hline & P 9 & Rosa & Rosa & Rosa & $=$ & $=$ \\
\hline
\end{tabular}

$\mathrm{P}=$ participante; $\uparrow$ indica aumento; $\downarrow$ significa redução; = indica que não houve alteração; - não ocorreu reavaliação. As cores e valores de força de cada filamento são: verde $(0,05 \mathrm{~g})$, azul $(0,2 \mathrm{~g})$, violeta $(2,0 \mathrm{~g})$, vermelho $(4,0 \mathrm{~g})$, laranja $(10,0 \mathrm{~g})$ e rosa $(300,0 \mathrm{~g})$. 
Os resultados do presente estudo demonstraram uma significante melhora dos pacientes nos quatro domínios avaliados bem como no escore total quando comparado a avaliação inicial $(p<0,05)$ (Figura 4).

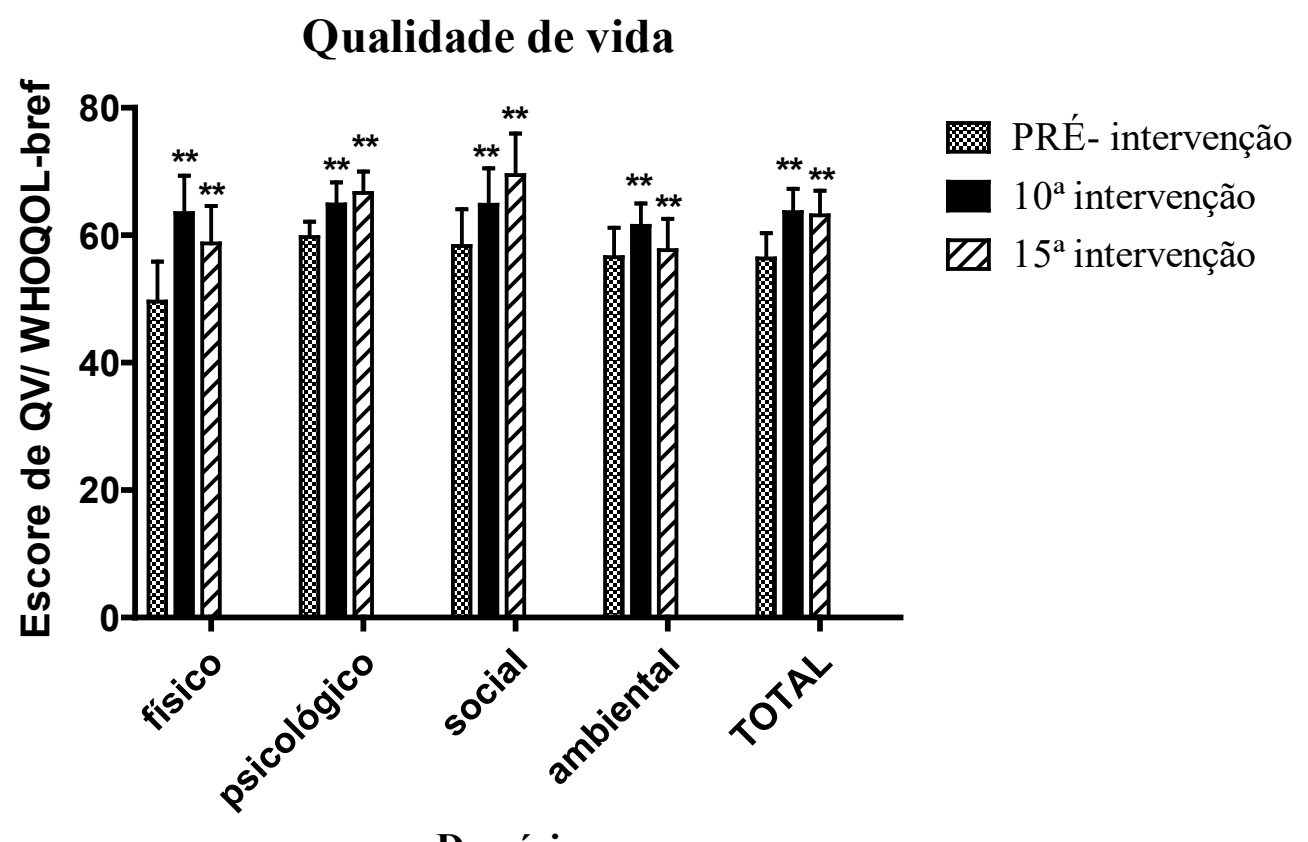

Figura 4- Escores dos domínios de qualidade de vida. Valores representados em média \pm erro padrão da média. ${ }^{* *}=$ diferença significativa em comparação a pré-intervenção $(P<0,05)$.

\section{DISCUSSÃO}

Os achados deste estudo mostram que em indivíduos com úlceras cutâneas, mesmo de diferentes etiologias, o emprego da fotobioestimulação e da estimulação elétrica pode ser considerado uma opção útil e benéfica. Nossos resultados sugerem que a aplicação do laser de baixa potência e da microcorrente, de forma isolada ou associados, pode promover redução na área da ferida com consequente melhora na cicatrização. Além de, gerar ganhos na qualidade de vida (QV) desses pacientes, considerando aspectos físicos, psicológicos, sociais e ambientais.

É importante salientar que os resultados desta pesquisa indicam tendência à diminuição do tamanho da área da lesão cutânea, com o tempo maior de intervenções. Ainda, a melhora na qualidade de vida foi confirmada pelo aumento em todos os escores nos domínios avaliados, na $10^{\mathrm{a}}$ e $15^{\mathrm{a}}$ intervenções evidenciando os benefícios dos recursos terapêuticos utilizados.

A mensuração das feridas por meio de métodos utilizando a régua de papel descartável e/ou através das fotografias digitais é essencial para o acompanhamento da evolução da cicatrização ${ }^{15}$. A redução na área (comprimento e 
largura) da úlcera cutânea observada nesta pesquisa, condizem com outros achados que também verificaram uma ferida de tamanho menor relacionada ao aumento na taxa de cicatrização após o uso do laser de baixa potência19 e da microcorrente ${ }^{7}$.

Pesquisa recente desenvolvida por Mathur e colaboradores (2017), com pacientes diabéticos, aponta que 0 laser de baixa potência é uma modalidade eficaz para facilitar a contração da ferida bem como na melhora da granulação tecidual, podendo esse recurso ser usado como um complemento ao modo convencional de tratamento ${ }^{21}$. Também, é importante observar que, a terapia com laser vermelho de baixa intensidade promove redução do processo inflamatório concomitante ao aumento na síntese de colágeno com consequente aumento da resistência tênsil da pele e favorecimento a resposta de reparo tecidual ${ }^{22}$. Do mesmo modo, Nair (2018) em recente ensaio clínico randomizado com pacientes queimados, destacou-se o uso da microcorrente, uma vez que a utilização desse recurso, acarretou em redução da área da ferida acelerando a cicatrização ${ }^{23}$. Ainda, pacientes tratados com essa forma de estimulação elétrica apresentaram diminuição na área da ferida bem como na dor, além de redução da sintomatologia inflamatória juntamente a melhora na qualidade do sono, na marcha e na frequência de movimento ${ }^{24}$. Salienta-se que, os efeitos benéficos da microcorrente podem ser oriundos do restabelecimento da bioeletricidade tecidual, promovido por essa corrente, causando aumento da velocidade de contração e fechamento da ferida ${ }^{12}$.

Ao mesmo tempo, ao analisar a terapia com microcorrente associada ao laser de baixa potência e intensidade na cicatrização por segunda intenção em ratos, Trombini e colaboradores (2015) constataram em análise histológica que a associação desses recursos terapêuticos provocou um aumento da área de colágeno ${ }^{25}$. Assim nossos achados corroboram com a afirmativa acima, uma vez que houve uma redução na área da úlcera cutânea quando os recursos foram aplicados de forma isolada bem como quando houve associação dos mesmos. Porém, Freitas et al. ${ }^{26}$, reportam a ideia de que a combinação do laser e microcorrente diminui a ação terapêutica desses dois recursos, promovendo redução dos efeitos do tratamento quando comparado ao uso dessas modalidades de forma isolada. $\mathrm{O}$ estudo desenvolvido por eles utilizou a laserterapia e micrococorrente na cicatrização de queimadura em ratos Wistar e concluiu que a associação dos recursos promoveu melhora apenas na neoangiogênese, já quando aplicados separadamente, aceleraram todas as fases da cicatrização. Neste contexto, pode-se sugerir que essa diferença nos achados esteja relacionada aos diferentes modelos experimentais utilizados e a etiologia das feridas tratadas.

Dessa forma, a hipótese de que a abordagem fisioterapêutica poderia melhorar a área de superfície da lesão e consequente taxa de cicatrização foi confirmada na amostra deste estudo. É fundamental ressaltar que a escolha de parâmetros como dose, tempo e comprimento de onda são de grande relevância para os efeitos benéficos da terapia com laser de baixa potência sendo que doses compreendidas entre 3-6 $\mathrm{J} / \mathrm{cm}^{2}$ mostram-se mais eficazes e não estão associadas a efeitos deletérios, ainda os comprimentos de onda entre 632,8-1000nm garantem melhores resultados 
no processo de cicatrização ${ }^{27}$. Do mesmo modo, salienta-se que na utilização da estimulação elétrica por meio da microcorrente visando-se o reestabelecimento tecidual, uma intensidade em microampères e tempo de tratamento longo (várias semanas, por horas e diariamente) implicaram em bons resultados na pratica clínica ${ }^{11}$. Entretanto, Ud-Din e colaboradores em 2014 relataram que há diferenças contrastantes nos parâmetros e tipos de aplicação da estimulação elétrica, levando a uma incapacidade de gerar evidências suficientes para apoiar um padrão de abordagem terapêutica ${ }^{6}$.

Outro aspecto importante a ser discutido são os resultados obtidos nas variáveis relacionadas à dor e à sensibilidade. Constatou-se que alguns pacientes apresentaram modificações no quadro álgico e na sensibilidade em resposta a aplicação dos equipamentos eletrotermofoterapêuticos. Os resultados com a EVA demonstraram que o laser e a microcorrente apresentam tendência à redução do quadro álgico dos indivíduos na $10^{\mathrm{a}}$ e $15^{\mathrm{a}}$ aplicação. Enwemeka e colaboradores (2004) evidenciaram mediante a realização de metanálise que a fototerapia por meio do laser de baixa potência é altamente efetiva para o reparo tecidual e alívio da dor ${ }^{28}$. Além disso, ao realizar um estudo-piloto para um ensaio clínico avaliando-se o efeito da estimulação elétrica, por microcorrente, sobre a dor e a área de superfície de úlceras venosas durante 4 semanas de tratamento, Korelo e colaboradores (2012) verificaram uma redução do limiar de dor, bem como uma significativa diminuição na área da ferida de pacientes com úlceras venosas ${ }^{29}$.

Uma provável explicação para os resultados referentes a dor e sensibilidade verificados em nosso estudo é a diferença no tempo da reparação tissular bem como na profundidade das úlceras, uma vez que são fatores que podem influenciar nesses aspectos clínicos. Neste sentido, Gardner e colaboradores em 2017, relataram que mecanismos biológicos como baixos níveis de oxigênio e redução do pH na ferida promovem a sensibilização de nociceptores resultando em um quadro álgico mais exacerbado; assim como, níveis mais elevados de citocinas inflamatórias, presentes na fase inflamatória e a úlcera ser ou tornar-se mais superficial, também podem estar associados ao aumento da dor ${ }^{30}$.

Entre os principais achados deste estudo, destaca-se que o uso do laser de baixa potência e da microcorrente em úlceras cutâneas, com 10 aplicações, durante 30 dias, em média, melhorou significativamente todos domínios avaliados (físico, psicológico, social e ambiental). É interessante enfatizar que avaliar a QV é tão importante quanto o cuidado da ferida, e os fatores clínicos influenciam diretamente os domínios de QV, comprometendo-os, todavia esses podem ser modificados conforme a execução de um tratamento eficaz ${ }^{4}$.

Sugere-se que esses equipamentos sejam utilizados nas clínicas de reabilitação bem como nos Serviços públicos, considerando que o custo dos mesmos é relativamente baixo e podem contribuir na recuperação de tecidos ${ }^{25}$. É importante ressaltar que a melhora na qualidade de vida dos pacientes é um dos principais objetivos de todos dos profissionais de saúde. 


\section{CONSIDERAÇÕES FINAIS}

Diante das condições experimentais realizadas, pode se verificar que as áreas de todas as úlceras cutâneas estimuladas com o laser e/ou microcorrente reduziram e houve cicatrização completa em duas delas. Desse modo, conclui-se que ambas as terapêuticas associadas ou não facilitaram o processo de cicatrização e melhoraram a qualidade de vida dos pacientes. Esses achados contribuem para a prática clínica uma vez que é importante avaliar os efeitos de recursos fisioterapêuticos não-invasivos e indolores, quando associados ou não, afim de melhorar a terapêutica utilizada em indivíduos com úlceras cutâneas em benefício da qualidade de vida dos mesmos.

\section{AGRADECIMENTOS}

Agradecemos a Equipe de enfermagem do Ambulatório B do Hospital Universitário de Santa Maria e aos pacientes com úlceras cutâneas que contribuíram para a realização deste estudo.

\section{REFERÊNCIAS}

1. Martin P, Nunan R. Cellular and molecular mechanisms of repair in acute and chronic wound healing. $\mathrm{Br} \mathrm{J}$ Dermatol. 2015; 173(2):370-8. doi: 10.1111/bjd.13954

2. Driver VR, Gould LJ, Dotson P, Allen LL, Carter MJ, Bolton LL. Evidence supporting wound care end points relevant to clinical practice and patients' lives. Part 2. Literature survey. Wound Repair Regen. 2019; 27(1):80-9. doi: 10.1111/wrr.12676

3. Lindholm C, Searle R. Wound management for the 21st century: combining effectiveness and efficiency.Int Wound J.2016; 13(Suppl 2):5-15. doi: 10.1111/iwj.12623

4. Oliveira AC, Rocha DM, Bezerra SM, Andrade EM, Santos AM, Nogueira LT. Qualidade de vida de pessoas com feridas crônicas. Acta Paul Enferm. 2019;32(2):194-201. doi: 10.1590/1982-0194201900027

5. Aldunate JL, Isaac C, Ladeira P, Carvalho V, Ferreira M. Venous ulcer in lower extremities. Revista De Medicina. 2010; 89(3-4), 158-63. doi: 10.11606/issn.1679-9836.v89i3/4p158-163 
6. Ud-Din S, Bayat A. Electrical Stimulation and Cutaneous Wound Healing: A Review of Clinical Evidence. Healthcare (Basel). 2014; 2(4):44567.doi:10.3390/healthcare20404

45

7. Martelli A, Theodoro V, Zaniboni VE, Freitas BA, Pastre GM, Melo KM, et al. Microcorrente no processo de cicatrização: revisão da literatura. Arch Health Invest. 2016, 5 (3): 134-9. doi: 10.21270/archi.v5i3.1316

8. Carrinho PM, Andreani DIK, Morete VA, Iseri S, Navarro RS, Villaverde AB.. A Study on the Macroscopic Morphometry of the Lesion Area on Diabetic Ulcers in Humans Treated with Photodynamic Therapy Using Two Methods of Measurement. Photomed Laser Surg. 2018; 36(1):44-50. doi: 10.1089/pho.2017.4305

9. Hamblin MR. Mechanisms and Mitochondrial Redox Signaling in Photobiomodulation. Photochem Photobiol. 2018; 94(2):199-212. doi: 10.1111/php.12864

10. Okuni I. Phototherapy in rehabilitation medicine. Masui. 2012; 61(7):700-5.

11. Poltawski L, Watson T. Bioelectricity and microcurrent therapy for tissue healing. Phys Ther Rev 2009; 14(2):104-14. doi: 10.1179/174328809X405973

12. Badhe RV, Nipate SS. Low-intensity current (LIC) stimulation of subcutaneous adipose derived stem cells (ADSCs) - A missing link in the course of LIC based wound healing. Med Hypotheses. 2019; 125:79-83. doi: 10.1016/j. mehy.2019.02.039.

13. Portney LG, Watkins MP. Foundations of Clinical Research Applications to Practice. 3rd Edition; 2015.

14. Ministério da Saúde (Brasil) Secretaria de Políticas de Saúde. Departamento de Atenção Básica. Manual de

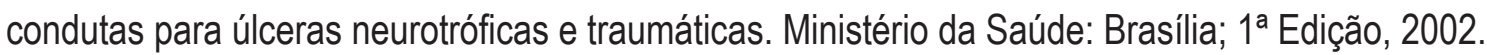

15. Eberhardt TD, Kessler M, Soares RSA, Lima SBS, Fonseca GGP, Rodrigues LR. Mensuração de feridas: revisão da literatura. Ciências \& Saúde. 2015; 8(2):79-84. doi: 10.15448/1983-652X.2015.2.19947 
16. Wewers ME, Lowe NK. A critical review of visual analogue scales in the measurement of clinical phenomena. Res Nurs Health.1990; 13(4):227-36.

17. The WHOQOL Group. The World Health Organization Quality of Life assessment (WHOQOL): position paper from the World Health Organization. Soc Sci Med. 1995; 41(10):1403-9. doi: 10.1016/0277-9536(95)00112-K

18. Fleck MPA, Louzada S, Xavier M, Chachamovich E, Vieira G, Santos L et al. Aplicação da versão em português do instrumento abreviado de avaliação da qualidade de vida "WHOQOL-bref". Rev. Saúde Pública, 2000; 34 (2):178-83, 2000. doi: 10.1590/S0034-89102000000200012

19. Feitosa MCP, Carvalho AFM, Feitosa VC, Coelho IM, Oliveira RA, Arisawa EAL. Effects of the Low-Level Laser Therapy (LLLT) in the process of healing diabetic foot ulcers. Acta Cirur. Bras. 2015; 30(12):852-7.doi: 10.1590/ S0102-865020150120000010

20. Balakatounis KC, Angoules AG. Low-intensity electrical stimulation in wound healing: review of the efficacy of externally applied currents resembling the current of injury. Eplasty. 2008; 8(e28): 283-91.

21. Mathur RK, Sahu K, Saraf S, Patheja P, Khan F, Gupta PK. Low-level laser therapy as an adjunct to conventional therapy in the treatment of diabetic foot ulcers. Lasers Med Sci. 2017; 32(2):275-82. doi: 10.1007/s10103-016-2109-2

22. Carvalho KC, Nicolau RA, Maia ALM, Barja PR, de Sá HP, Santo LAE et al. Estudo da resistência cicatricial cutânea de ratos tratados com fototerapia a laser. ConScientiae Saúde. 2010; 9(2):179-86. doi: 10.5585/conssaude. v9i2.2267

23. Ibrahim ZM, Waked IS, Ibrahim O. Negative pressure wound therapy versus microcurrent electrical stimulation in wound healing in burns. J Wound Care. 2019; 28(4):214-9. doi: 10.12968/jowc.2019.28.4.214

24. Nair HKR. Microcurrent as an adjunct therapy to accelerate chronic wound healing and reduce patient pain. J Wound Care. 2018; 27(5):296-306. doi: 10.12968/jowc.2018.27.5.296 
25. Trombini KCB, Costa DR, Nicolau RA. Efeitos do laser GaAlAs associado à microcorrentes na cicatrização por segunda intenção. ConScientiae Saúde. 2015;14(4):532-40. doi:10.5585/ConsSaude.v14n4.5752

26. Freitas RPA, Barcelos APM, Nóbrega BM, Macedo AB, Oliveira AR, Ramos AMO et al. Low-level lasertherapy and micro current in burn wound healing in rats. Associated or isolated therapy? Fisioter Pesq. 2013;20(1):24-30.

27. Andrade FSS, Clark RMO, Ferreira, ML. Effects of low-level laser therapy on wound healing. Re. Col Bras Cir. 2014;41(2):129-33. doi:10.1590/S0100-69912014000200010

28. Enwemeka CS, Parker JC, Dowdy DS, Harkness EE, Sanford LE, Woodruff LD. The efficacy of low-power lasers in tissue repair and pain control: a meta-analysis study. Photomed Laser Surg. 2004;22(4):323-9. doi: 10.1089/ pho.2004.22.323

29. Korelo RIG, Valderramas S, Ternoski B, Medeiros DS, Andres LF, Adolph SMM. Aplicação da microcorrente como recurso para tratamento de úlceras venosas: um estudo piloto. Rev. Latino-Am. Enfermagem [Internet]. 2012; 20(4):753-60.

30. Gardner SE, Abbott LI, Fiala CA, Rakel BA. Factors associated with high pain intensity during wound care procedures: A model. Wound Repair Regen. 2017; 25(4):558-563. doi: 10.1111/wrr.12553 